\title{
Qualität in der ambulanten Medizin: Zusammen ist man stärker
}

\author{
Vier Organisationen, die sich mit der Qualitätsmessung und -förderung in Arztpra- \\ xen befassen, starten anlässlich des Symposiums in Luzern unter dem Motto \\ «Q-Initiative» einen gemeinsamen Auftritt. Sie wollen die Kultur der Versorgungs- \\ qualität im ambulanten Gesundheitsbereich stärken und Anreize schaffen, damit \\ sich Qualitätsbemühungen für die Arztpraxis lohnen.
}

Ignazio Cassisa, Wolfgang Czerwenka ${ }^{b}$, Christoph Ramstein', Alexander von Weymarn $^{d}$

a Dr. med., Nationalrat, Präsident EQUAM Stiftung (Externe Qualitätssicherung in der ambulanten Medizin) Q-Module

b Dr. med., med. GL, Argomed Ärzte AG - MFA (Mehrfacharzt); Schweiz. Dienstleistungsunternehmen für med. Versorgungsmodelle und Ärztenetze

c Dr. med., Präsident medswiss.net - QMN (quali-med-net), Schweizer Dachverband der Ärztenetze

d Dr. med, CO-Präsident VEDAG - QBM (QualitätsBasis-Modul), Verband deutschschweizerischer Ärztegesellschaften

Korrespondenz: Sigrid Hess-Scheurer, MSC EQUAM Stiftung Effingerstrasse 25 CH-3008 Bern Tel. 0612710111 sigrid.hess[at]equam.ch
Folgende vier schweizerische Organisationen haben sich für den gemeinsamen Auftritt auf dem Symposium in Luzern zusammengetan: die EQUAM Stiftung, der Schweizerische Dachverband der Ärztenetze medswiss.net, die Argomed Ärzte AG sowie der Verband deutschschweizerischer Ärztegesellschaften VEDAG (s. Abb. nächste Seite).

Seit dem Inkrafttreten des Krankenversicherungsgesetzes im Jahr 1996 sind Nachweis und Förderung der Qualität explizit in Art. 58 gefordert, dies als gemeinsame Arbeit der Tarifpartner (Leistungserbringer und Krankenversicherer). Leider konnte bis heute das Gesetz Leistungserbringer (Arztpraxen, Gruppenpraxen und integrierte Versorgungsnetze), die sich pro-aktiv für die Qualität einsetzen, nicht belohnen, und Anreize wurden keine geschaffen. aktiv zu werden. Sie alle befassen sich seit Jahren mit der Qualitätsentwicklung. Da jede einzelne Organisation für sich allein zu wenig durchschlagkräftig ist, um auf das ganze ambulante Gesundheitssystem eine Wirkung zu erzielen, haben die vier Organisationen ihre Kräfte gebündelt. Der gemeinsame Auftritt soll zu mehr Sensibilität bezüglich Qualitätsarbeit in den Arztpraxen führen und Anreize schaffen, Qualitätssysteme zur Sicherung einer hochqualitativen medizinischen Versorgung einzuführen.

Die mit einem Qualitätslabel ausgezeichneten Arztpraxen erfüllen nachweislich definierte Qualitätsanforderungen, die durch externe Evaluation überprüft und damit für Patienten, Behörden, Versicherungen und weitere Kreise transparent gemacht werden. Das Erreichen von Qualitätsanforderungen

\section{«Mit Teilnahmebestätigungen, Qualitätslabels oder sogar Zertifizierungen kann die Qualität evident gemacht werden.»}

Da die Tarifpartner - gemäss Aussage des schweizerischen Parlamentes - sich nur ungenügend um Qualitätsfragen gekümmert haben, hat dieses 2005 den Bundesrat beauftragt, die Führung im Bereich Qualität der Gesundheitsversorgung zu übernehmen. Seitdem arbeitet das Bundesamt für Gesundheitswesen, zusammen mit allen Gesundheitspartnern, an der Konkretisierung einer nationalen Qualitätsstrategie.

Die vier Organisationen aus dieser Q-Initiative haben nicht auf die nationale Strategie gewartet, um und Minimalstandards erhöht das Vertrauen in die entsprechenden Leistungserbringer. Mit Teilnahmebestätigungen, Qualitätslabels oder sogar Zertifizierungen kann die Qualität evident gemacht werden. Eine Übersicht über die Qualitätslabels der oben erwähnten Institutionen finden Sie in der beigelegten Zusammenstellung.

Mit dem gemeinsamen Auftritt anlässlich des Symposiums Managed Care und Integrierte Versorgung vom 7. Februar 2013 wird die Q-Initiative vorgestellt und lanciert. 


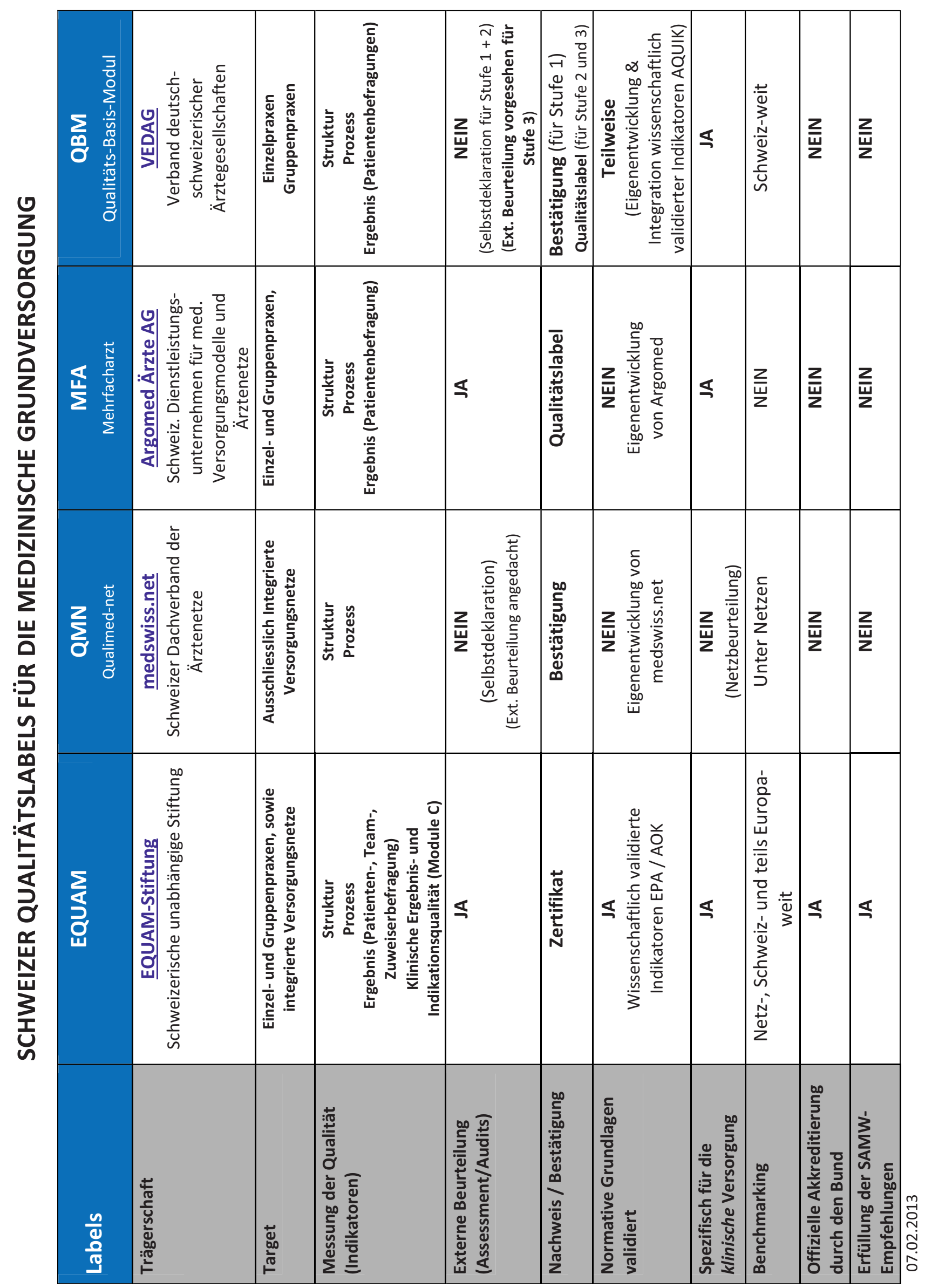

